\title{
Oleato de monoetanolamina 5\% como tratamento para hemangioma de infância: Um relato de experiência
}

\author{
Monoetanolamine oleate 5\% as a treatment for childhood hemangioma: An experience report \\ Oleato de monoetanolamina $\mathbf{5 \%}$ como tratamiento del hemangioma infantil: Informe de
} experiencia

\author{
Anne Diollina Araújo Morais \\ ORCID: https://orcid.org/0000-0002-7208-5520 \\ Universidade Federal do Ceará, Brasil \\ E-mail: annemorais85@gmail.com \\ Carlos Eduardo Nogueira Nunes \\ ORCID: https://orcid.org/0000-0003-4223-4633 \\ Universidade Federal do Ceará, Brasil \\ E-mail: caduunogueiraa@gmail.com \\ Irla Maria Sousa Moura \\ ORCID: https://orcid.org/0000-0003-0990-2281 \\ Universidade Federal do Ceará, Brasil \\ E-mail: irlamaria1258@hotmail.com \\ Filipe Nobre Chaves \\ ORCID: https://orcid.org/0000-0001-6345-8156 \\ Universidade Federal do Ceará, Brasil \\ E-mail: filipenobrechaves@hotmail.com \\ Marcelo Bonifácio da Sila Sampieri \\ ORCID: https://orcid.org/0000-0001-7942-6642 \\ Universidade Federal do Ceará, Brasil \\ E-mail:mar_sampieri@hotmail.com \\ Denise Hélen Imaculada Pereira de Oliveira \\ ORCID: https://orcid.org/0000-0001-6020-2374 \\ Universidade Federal do Ceará, Brasil \\ E-mail: denise.oliveira@sobral.ufc.br
}

\section{Resumo}

As anomalias vasculares da região de cabeça e pescoço representam um grupo complexo de lesões de origem congênita ou adquirida, tendo o hemangioma de infância (HI) como o tumor vascular benigno mais comum da infância. O HI é caracterizado por uma fase de crescimento rápido seguida de uma fase involuída, presente geralmente antes do primeiro ano de vida. A escleroterapia é um dos tratamentos padrões para esse tipo de anomalia, mesmo que ainda não exista um protocolo padronizado para esse fim. Com isso, considerando os dados de eficácia e segurança da escleroterapia com oleato de monoetanolamina (OM) relatados na literatura, o objetivo deste estudo é relatar o uso do OM de 5\% em pacientes com HI. Trata se de um estudo descritivo, com abordagem qualitativa, do tipo relato de experiência, com injeções intralesionais de aproximadamente $0,1 \mathrm{ml}$ de $\mathrm{OM}$ de $5 \%$ por $4 \mathrm{~mm}$ de lesão com intervalo de 14 dias entre as sessões. Como resultado, obteve se resolução completa de todos os quatro casos aplicados com duas sessões de injeção intralesional de OM de 5\%. A escleroterapia com OM é uma opção de tratamento acessível e aceitável que mostrou eficácia quando usada adequadamente, porém sendo de suma importância a execução de um tratamento individual e personalizado para cada caso.

Palavras-chave: Escleroterapia; Hemangioma; Terapêutica.

\begin{abstract}
Vascular anomalies in the head and neck represent a complex group of lesions of congenital or acquired origin, with childhood hemangioma (HI) as the most common benign vascular tumor in childhood. HI is characterized by a phase of rapid growth followed by an involuted phase, usually present before the first year of life. Sclerotherapy is one of the standard treatments for this type of anomaly, even though there is still no standardized protocol for this purpose. Thus, considering the efficacy and safety data of sclerotherapy with monoethanolamine oleate (OM) reported in the literature, the aim of this study is to report the use of 5\% OM in patients with HI. This is a descriptive study, with a qualitative approach, of the experience report type, with intralesional injections of approximately $0.1 \mathrm{ml}$ of $5 \%$ OM for $4 \mathrm{~mm}$ of lesion with an interval of 14 days between sessions. As a result, complete resolution of all four cases was obtained with two sessions of intralesional injection of OM of 5\%. Sclerotherapy with OM is an affordable and
\end{abstract}


acceptable treatment option that has proven effective when used properly, but it is extremely important to carry out an individual and personalized treatment for each case.

Keywords: Sclerotherapy; Hemangioma; Therapy.

\section{Resumen}

Las anomalías vasculares en la cabeza y el cuello representan un grupo complejo de lesiones de origen congénito o adquirido, siendo el hemangioma infantil $(\mathrm{IH})$ el tumor vascular benigno más común en la infancia. La HI se caracteriza por una fase de crecimiento rápido seguida de una fase involucionada, generalmente presente antes del primer año de vida. La escleroterapia es uno de los tratamientos estándar para este tipo de anomalías, aunque todavía no existe un protocolo estandarizado para tal fin. Así, considerando los datos de eficacia y seguridad de la escleroterapia con oleato de monoetanolamina (MO) reportados en la literatura, el objetivo de este estudio es reportar el uso de $\mathrm{MO}$ al $5 \%$ en pacientes con IH. Se trata de un estudio descriptivo, con abordaje cualitativo, del tipo de relato de experiencia, con inyecciones intralesionales de aproximadamente $0,1 \mathrm{ml} \mathrm{de} \mathrm{MO}$ al $5 \%$ por $4 \mathrm{~mm}$ de lesión con un intervalo de 14 días entre sesiones. Como resultado, se logró la resolución completa de los cuatro casos con dos sesiones de inyección intralesional de $\mathrm{OM}$ al 5\%. La escleroterapia con OM es una opción de tratamiento asequible y aceptable que ha demostrado su eficacia cuando se utiliza correctamente, pero es de suma importancia realizar un tratamiento individual y personalizado para cada caso.

Palabras clave: Escleroterapia; Hemangioma; Terapia.

\section{Introdução}

Anomalias vasculares (AVs) são lesões de origem congênita ou adquirida cujos componentes predominantes são estruturas vasculares (Enjoras, Wassef, \& Chapot, 2013). As AVs incluem uma diversidade de tumores e malformações que variam de simples irregularidades capilares à estruturas complexas envolvendo artérias, veias e vasos linfáticos; sendo o HI o tumor vascular benigno mais comum da infância (Fernandes et al.,2018; Brahmbhatt, Skalski, \& Bhatt, 2020; ISSVA, 2018).

O diagnóstico clínico do HI é baseado sem suas características clínicas e no seu histórico, tendo a vitropressão e os exames de imagem como complementares que auxiliam no diagnóstico e em um tratamento mais precisos (Fernandes et al.,2018). Além disso, achados importantes, como a hemodinâmica da lesão (fluxo sanguíneo alto ou baixo) devem ser considerados na prática do tratamento (Enjoras, Wassef, \& Chapot, 2013; Baer et al., 2011). Apesar de sua natureza benigna, o HI pode causar desconforto estético, dor, ulceração, sangramento, infecção secundária, deformação do tecido, comprometimento da fala e obstrução das vias áreas superiores (Greene, 2012; Sigaux et al., 2015).

A excisão cirúrgica é um dos tratamentos mais utilizados, principalmente para pequenas lesões (Johann et al., 2005; Mariano et al., 2011). Porém, antes da escolha desta modalidade de tratamento, alguns critérios devem ser considerados, como sangramento, ressecção incompleta da lesão e problemas estéticos (Johann et al., 2005; Mariano et al., 2011). Métodos alternativos são mencionados na literatura, incluindo cirurgia a laser, crioterapia, quimioterápicos, corticosteroides, embolização e escleroterapia (Johann et al., 2005; Corrêa et al., 2007; Sadick et al., 2017).

A escleroterapia é classificada como uma técnica eficaz e minimamente invasiva, proporcionando taxas de cicatrização de $70 \%$ a 100\% que excluem a necessidade de uma intervenção cirúrgica (Bonam et al., 2007; Fernandes et al., 2018; Kato et al., 2019). Embora muitos agentes esclerosantes estejam disponíveis no mercado, o oleato de monoetanolamina $(\mathrm{OM})$ é particularmente útil devido à sua baixa toxicidade em comparação com outros agentes indutores de esclerose (Johann et al., 2005; Costa et al., 2011). Estudos prévios mostraram a eficácia do OM em diferentes concentrações (1,25\%, 2,5\% e 5\%), números de sessões e volume injetado para o tratamento das AVs orais (Johann et al., 2005; da Silva et al., 2014; Fernandes et al., 2018; Kato et al., 2019). Com isso, a concentração ideal de OM para tratar AVs orais ainda não foi estabelecida (Kato et al.,2020).

Considerando os dados de eficácia e segurança na escleroterapia com OM relatado na literatura, o objetivo deste estudo é relatar o uso do OM de $5 \%$ em pacientes com HI. 


\section{Metodologia}

Este artigo trata se de um estudo qualitativo, descritivo, retrospectivo, do tipo relato de experiência (Pereira et al., 2018). Este trabalho foi submetido e aprovado pelo Comitê de Ética em Pesquisa da Universidade Estadual do Vale do Acaraú - CE sob o número de parecer 4.206.372 e CAAE 34474920.4.0000.5053.

Quatro pacientes foram incluídos nesta coorte. Todos os pacientes foram tratados no Ambulatório de Estomatologia da Universidade Federal do Ceará - campus Sobral. O diagnóstico clínico de HI foi realizado através da história clínica da lesão e vitropressão. As indicações para tratamento incluíram inchaço, crescimento e queixas estéticas. Todos os pacientes foram tratados com OM de 5\% (Ethamolin ${ }^{\circledR}$ ).

O protocolo de injeção intralesional consistiu em aproximadamente $0,1 \mathrm{ml}$ de OM de $5 \%$ por $4 \mathrm{~mm}$ de lesão, tendo como base o uso seguro de OM de 5\% não diluído em uma dose de $0,1 \mathrm{ml}$ por $3 \mathrm{~mm}$ de lesão com intervalo de 14 dias entre as sessões (Kato et al.,2019) e o limite máximo da dose de OM de $20 \mathrm{ml}$ em pacientes adultos ou 0,3 $\mathrm{ml} / \mathrm{kg}$ (Ozaki et al., 2010).

Os HIs foram primeiramente avaliados e mensurados para determinar o volume da injeção intralesional. A droga foi injetada com uma seringa de insulina com agulha curta. Paciente retornou após 7 dias para avaliação e com 14 dias para segunda aplicação. Um único operador realizou as injeções.

\section{Resultados}

Um total de quatro pacientes foram atendidos com diagnóstico clínico de HI após análise da história clínica da lesão e vitropressão positiva. Três pacientes eram do sexo masculino e um do sexo feminino, com idades entre 37 a 70 anos. As lesões variaram com tamanhos de $7 \mathrm{~mm}$ à $14 \mathrm{~mm}$ e tendo o lábio como local mais acometido, sendo presente em três casos.

Foi realizado uma injeção intralesional de aproximadamente $0,1 \mathrm{ml}$ de $\mathrm{OM}$ de $5 \%$ por $4 \mathrm{~mm}$ de lesão sendo aplicado em quatro partes. $\mathrm{O}$ volume final da droga variou entre $0,2 \mathrm{ml}$ e $0,4 \mathrm{ml}$. Foi observada uma regressão significativa na primeira sessão em apenas dois casos (Figura 1 e 2). 
Figura 1. Aspecto intraoral da lesão. (A) Aspecto clínico inicial de lesão em lábio superior; (B) Vitropressão positiva; (C) Injeção intralesional de OM de 5\% com seringa de insulina e agulha curta; (D) Aspecto clínico imediatamente após primeira aplicação; (E) Resultado após 15 dias da primeira aplicação de OM de 5\%; (F) Resultado final após 15 dias da segunda aplicação de OM de 5\%.

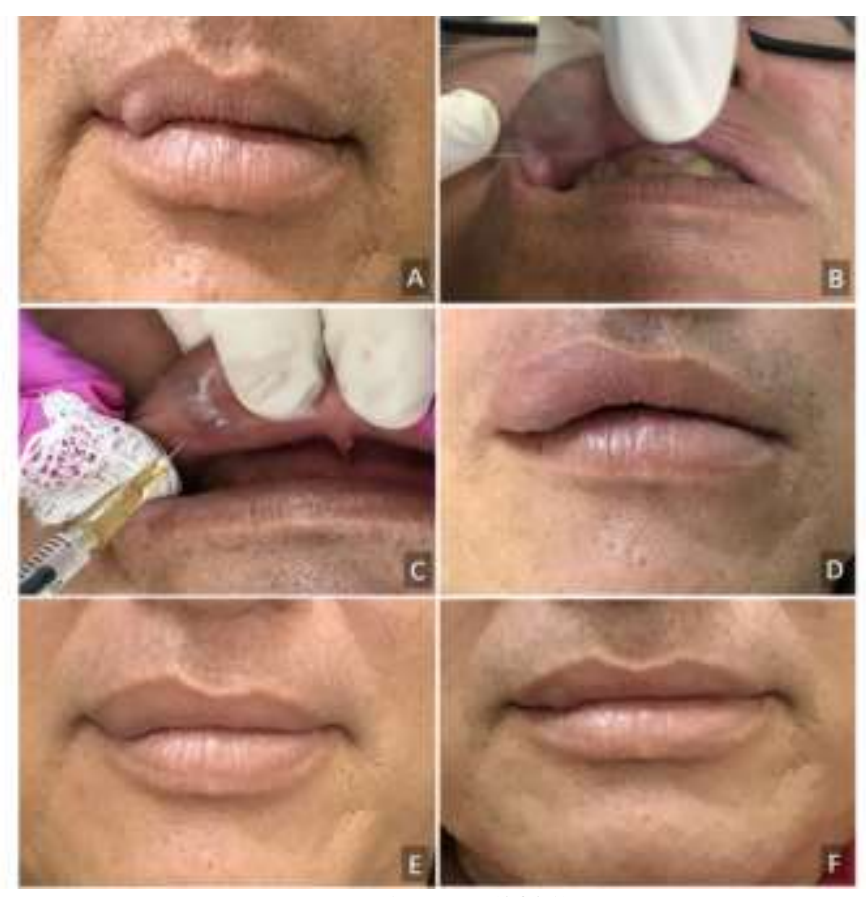

Fonte: Autores (2021).

Figura 2. Aspecto intraoral da lesão. (A) Aspecto clínico inicial de lesão em lábio inferior; (B) Aspecto clínico inicial de lesão em lábio inferior; (C) Injeção intralesional de OM de 5\% com seringa de insulina e agulha curta; (D) Aspecto clínico imediatamente após primeira aplicação com leve sangramento; (E) Resultado após 15 dias da primeira aplicação de OM de 5\%; (F) Resultado final após 15 dias da segunda aplicação de OM de 5\%.
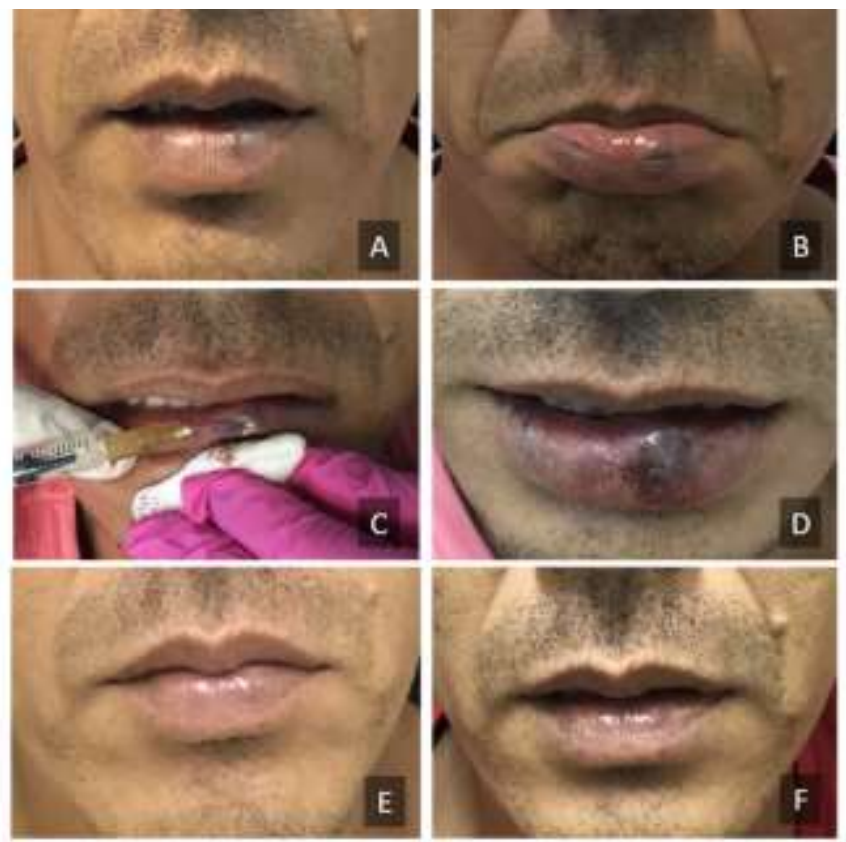

Fonte: Autores (2021). 
Todos os casos apresentaram resolução completa da lesão após duas sessões com intervalo de 14 dias entre elas (Figura 1, 2, 3 e 4). Edema e queimação após aplicações foram relatados pelos pacientes. Úlcera e hematoma foram observadas entre as sessões em dois casos, ambas com resolução espontânea (Figura 3). Também foi relatado endurecimento no local da antiga lesão em um caso (Figura 1).

Figura 3. Aspecto intraoral da lesão. (A) Aspecto clínico inicial de lesão em fundo de sulco; (B) Resultado após sete dias da primeira aplicação; (C) Hematoma após sete dias da segunda aplicação; (D) Resultado final após 15 dias da segunda aplicação de OM de $5 \%$.

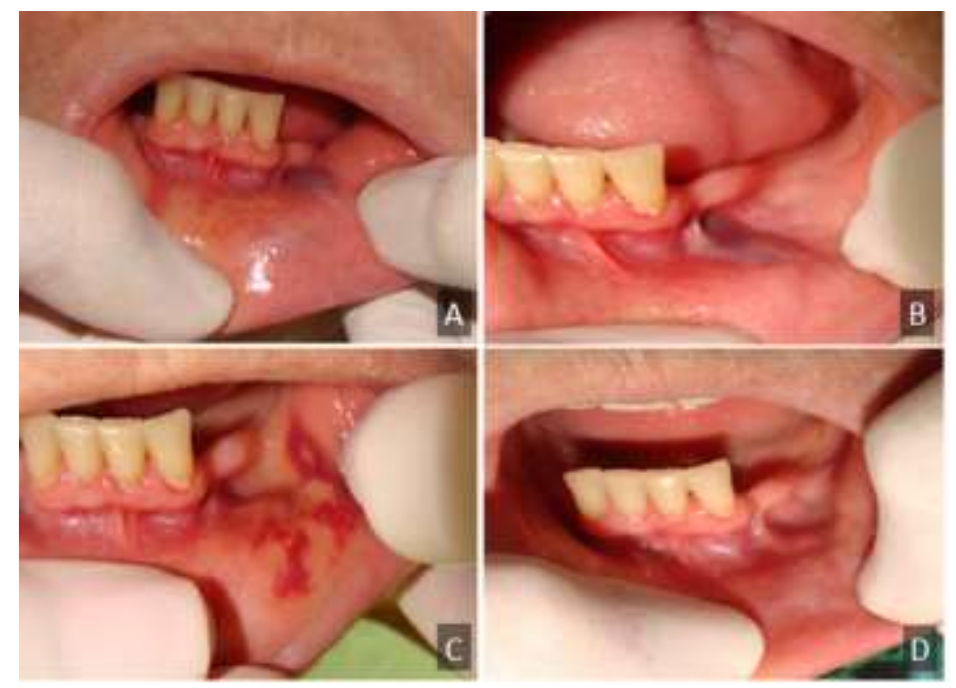

Fonte: Autores (2021).

Figura 4. Aspecto intraoral da lesão. (A) Aspecto clínico inicial de lesão em lábio inferior; (B) Resultado final após 15 dias da segunda aplicação de OM de 5\%.

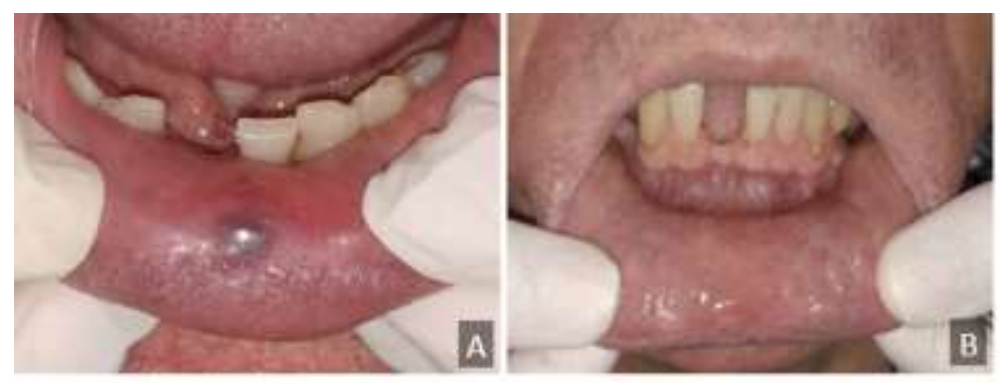

Fonte: Autores (2021).

\section{Discussão}

O HI pertence ao grupo de tumores vasculares proliferativos (ISSVA, 2018). O mesmo é o tumor vascular benigno mais comum da infância, visto em aproximadamente 5\% a 10\% das crianças, com predominância no sexo feminino (Eivazi \& Werner, 2013). A região de cabeça e pescoço é a sua localização de maior incidência (Kato et al.,2019). E, geralmente, o HI é percebido nos primeiros 8 dias de vida (Haggstrom et al., 2007; Eivazi \& Werner, 2013).

Clinicamente, a história natural do HI é dividida em três fases: fase inicial ou proliferativa, seguida da regressão gradual ou fase involutiva e, por fim, uma fase involuída (Ballah et al., 2011). A fase involuída do HI não implica 
obrigatoriamente em uma regressão total do tumor, uma vez que no local da lesão podem permanecer sequelas (Bruckner \& Frieden, 2003). Como neste estudo, em que todos os casos não apresentaram regressão completa da lesão de forma espontânea.

Embora o HI seja uma lesão benigna, eventualmente, sua história natural pode demonstrar complicações (Nair et al., 2018). A intervenção se torna ainda mais imprescindível após a fase de involução, particularmente na presença de excesso de tecido residual, cicatrização ou telangiectasia (Nair et al., 2018). Além disso, indicações claras de intervenção também são dadas quando sistemas vitais ou estruturas como vias aéreas ou visuais são afetadas (Nair et al., 2018).

O diagnóstico das lesões vasculares orais é baseado nas características clínicas e no histórico da lesão. A vitropressão e os exames de imagem também podem contribuir para o diagnóstico e o planejamento do tratamento em alguns casos (Baer et al., 2011). Neste estudo, a vitropressão foi realizada em todos os pacientes atendidos, tendo como resultado uma vitropressão positiva nos quatro casos, compatível com HI. Clinicamente, as lesões vasculares são geralmente assintomáticas, variando de alguns milímetros a vários centímetros em seu tamanho (Correa et al.,2007; Fernandes et al.,2018). A cor varia de vermelho a roxo, de acordo com a localização e profundidade da invasão tecidual, bem como quanto ao grau de congestão vascular da área afetada (Correa et al.,2007; Fernandes et al.,2018). Além disso, pode se apresentar como uma lesão plana ou elevada, com superfície lisa ou nodular, bordas definidas, sésseis ou pedunculadas e consistência suave à palpação (Correa et al.,2007; Fernandes et al.,2018). No presente estudo, todos os casos se apresentaram de forma assintomática, com uma superfície nodular e implantação séssil. Os tamanhos variaram de $7 \mathrm{~mm}$ à $14 \mathrm{~mm}$.

A escleroterapia vem sendo utilizada com grande sucesso sem a necessidade de intervenção cirúrgica, alcançando resultados clínicos e estéticos satisfatórios, sendo uma terapêutica viável e bem aceita pelo paciente (Kato et al., 2019). O tratamento da escleroterapia geralmente é realizado em casos com risco de sangramento e com comprometimento das funções estéticas e/ou fisiológicas, como fala e mastigação (Fernandes et al.,2018). Nesse contexto, algumas características importantes devem ser consideradas, incluindo idade do paciente, tamanho, localização e hemodinâmica da lesão (fluxo sanguíneo alto ou baixo) (Fernandes et al.,2018). A escolha do tratamento deve ser baseada principalmente nesses aspectos e na experiência do profissional (Fernandes et al.,2018). Três casos deste estudo apresentavam comprometimento estético e um caso comprometimento fisiológico. Todos os casos foram avaliados individualmente com base em suas características clínicas.

O mecanismo de ação da escleroterapia envolve a substituição do componente vascular por um tecido fibroso em resposta a um processo inflamatório gerado (Johann et al.,2005). Embora a escleroterapia seja um dos tratamentos mais versáteis e vantajosos, a concentração do agente esclerosante, a dose e o modo de aplicação ainda não são padronizados (Johann et al.,2005). A escleroterapia pode proporcionar resultados satisfatórios em lesões de vários tamanhos, principalmente em regiões de face e lábios onde outras opções de tratamento, como a cirurgia, podem comprometer a fisiologia e os aspectos estéticos finais (Mariano et al.,2011). Três dos nossos casos acometiam região de lábio, dois em lábio inferior e um em lábio superior.

Os agentes indutores de esclerose presentes no mercado são inúmeros, sendo o OM um agente esclerosante de baixo custo utilizado há mais de 60 anos e que, de acordo com a literatura, causa menos danos ao tecido conjuntivo em comparação com outros esclerosantes (Mariano et al., 2011; Johann et al., 2005; Costa et al., 2011). Além disso, os efeitos colaterais da OM são limitados e podem ser facilmente evitados quando usados adequadamente (Mariano et al., 2011).

O OM é um sal de uma gordura ácida insaturada que age como um agente esclerosante quando injetado intravenosamente (Johann et al, 2005). O mecanismo de ação do OM envolve uma resposta inflamatória intra e extravascular e uma fibrose do endotélio. O componente oleico provoca a coagulação local por meio da ativação do fator de Hagemman e a monoetanolamina inibe a formação do coágulo de fibrina pela quelação do cálcio (Ribas et al, 2004; Costa et al., 2011; Johann et al., 2005). A ação conjunta dessas duas substâncias permite um equilíbrio hemostático, evitando hemorragia após sua administração nas AVs e a substituição da lesão vascular por fibrose (Ribas et al, 2004; Costa et al., 2011; Johann et al., 2005). 
Estudos prévios mostraram a eficácia do OM em diferentes concentrações $(1,25 \%, 2,5 \%$ e 5\%), números de sessões e volume usado para o tratamento das AVs orais (Johann et al., 2005; da Silva et al., 2014; Fernandes et al., 2018; Kato et al., 2019). O número de sessões é variável, variando de 1 a 10 sessões (Johann et al.,2005; da Silva et al., 2014). Além disso, concentrações de OM de 1,25\% e 2,5\% podem resultar em um maior volume de líquido dentro da lesão, junto com maiores efeitos adversos repetidos devido à necessidade de mais sessões (Kato et al., 2019). Esses fatos são muitas vezes mal tolerados pelo paciente, que podem chegar a abandonar o tratamento (Kato et al., 2019). Além disso, Kato et al. (2020) em uma análise comparativa retrospectiva de pacientes com AVs tratados com OM mostrou que OM de 5\% é mais eficaz e seguro, e exige menos sessões de tratamento e um volume e dose finais menores. Assim, neste estudo foi utilizado OM de 5\% para reduzir a quantidade de sessões e, consequentemente, a frequência de exposição dos pacientes aos efeitos adversos da escleroterapia. Nos quatro casos foram necessárias apenas duas sessões com intervalo de 14 dias entre elas, assim, tendo uma resolução completa em até 30 dias, como no estudo de Kato et al. (2019), em que lesões maiores de 6mm tiveram uma remissão completa dentro de 30 dias.

Apesar da simplicidade e facilidade da técnica, a possibilidade de complicações associadas não devem ser ignoradas (Fernando et al.,2018). A incidência e a intensidade dessas complicações dependem diretamente da técnica utilizada, do tipo e da concentração do agente esclerosante e da experiência do profissional (Johann et al., 2005). Ulceração, sangramento, hematoma, edema e necrose são algumas das complicações locais que podem ocorrer após a aplicação de OM (da Silva et al., 2014; Costa et al., 2011). Um endurecimento no local antigo da lesão ao final do tratamento também pode ocorrer em alguns casos devido à uma produção exacerbada de tecido fibroso. Complicações sistêmicas, como reação anafilática, hemoglobinúria, lesão nervosa ou colapso cardiovascular pode ser observado nesse tipo de tratamento (Johann et al., 2005; Costa et al., 2011; Kaji et al., 2009). Neste estudo, foi observado hematoma e ulceração após aplicação em dois pacientes, um apresentou hematoma e outro ulceração, porém ambos com resolução espontânea. Além disso, um dos casos apresentou fibrose ao final do tratamento. $\mathrm{O}$ mesmo apresentava tendência à queloide e foi informado da possibilidade da formação desse tecido fibroso no local antigo lesão. Complicações sistêmicas não foram observadas neste estudo o que possivelmente se deve as baixas doses utilizadas nos quatro casos.

As contraindicações da escleroterapia e da técnica envolvem pacientes diabéticos não controlados, gestantes, lactantes e regiões com infecção secundária nas quais o tratamento pode causar edema e sangramento na área lesada (Fernandes et al., 2018). Neste estudo, nenhum paciente apresentava nenhum tipo de contraindicação para a utilização dessa terapia.

\section{Considerações Finais}

A escleroterapia com OM é uma opção de tratamento acessível e aceitável, mostrando eficácia quando usada adequadamente. Apesar de ainda não se ter um protocolo padrão a ser seguido, o uso dessa terapêutica deve ser encorajada por conta de sua segurança e eficácia. Embora dois casos deste estudo tenham exibido complicações locais, todas tiveram resolução espontânea e todos os pacientes ficaram satisfeitos com o resultado final do tratamento. Além disso, é de suma importância a execução de um tratamento individual e personalizado para cada caso.

A realização de mais estudos com o uso do OM para o tratamento de HI e de outros tipos de AVs, como ensaios clínicos randomizados, é de muita importância para que, assim, se tenha um maior número de estudos comprovando sua eficácia e o controle de possíveis efeitos adversos que venham a acontecer, assim, confirmando sua segurança de uso nesses casos. 


\section{Referências}

Baer, A. H., Parmar, H. A., Dipietro, M. A., Kasten, S. J., \& Mukherji, S. K. (2011). Hemangiomas and vascular malformations of the head and neck: A simplified approach. Neuroimaging Clin N Am, 21, 641-58.

Ballah, D., Cahill, A. M., Fontalvo, L., Yan, A., Treat, J., Low, D. \& Epelman M.(2011). Vascular anomalies: what they are, how to diagnose them, and how to treat them. Curr Probl Diagn Radiol., 6 (40), 233-247. 10.1067 / j.cpradiol.2011.04.001.

Bonan, P. R. F., Miranda, L. P., Mendes, D. C., de Paula, A. M. B., Pego, S. P. B., \& Júnior, H. M. (2007). Efferctives of low flow vascular lesions sclerosis with monoethanolamine: Report of six cases. Med Oral Patol Oral Cir Bucal., 12, 524-527. http://www.medicinaoral.com/pubmed/medoralv1 2_i7_pE524.pdf.

Brahmbhatt, A. N., Skalski, K. A., \& Bhatt, A. A. (2020). Vascular lesions of the head and neck: an update on classifcation and imaging review. Insights Imaging., 11, 19.10.1186/ s13244-019-0818-3.

Bruckner, A. L., \& Frieden, I. J. (2003) Hemangiomas of infancy. J Am Acad Dermatol, 48(3), 477-493. 10.1067 / mjd.2003.200.

Corrêa, P. H., Nunes, L. C., Johann, A. C. B. R., Aguiar, M. C. F., Gomez, R. S, \& Mesquita, R. A. (2007) Prevalence of oral hemangioma, vascular malformation and varix in a Brazilian population. Braz Oral Res, 21 (1), 40-45. 10.1590 / s1806-83242007000100007.

Costa, J. R. S., Torriani, M. A., Hosni, E. S., D'Avila, O. P., \& Figueiredo, P. J. (2011). Sclerotherapy for vascular malformations in the oral and maxillofacial region: treatment and follow-up of 66 lesions. J Oral Maxillofac Surg, 69, 88-92. 10.1016 / j.joms.2010.06.204.

Da Silva, W. B., Ribeiro, A. L., de Menezes, S. A. F., Pinheiro, J. J. V., \& Junior, S. M. A. (2014). Oral capillary hemangioma: a clinical protocol of diagnosis and treatment in adults. Oral Maxillofac Surg., 18 (4), 431-437. 10.1007 / s10006-013-0436-z.

Eivazi, B., \& Werner, J. A. (2013). Management of vascular malformations and hemangiomas of the head and neck - an update. Curr Opin Otolaryngol Head Neck Surg, 21, 157-163. 10.1097 / MOO.0b013e32835e15a9.

Enjoras, O., Wassef, M., \& Chapot, R. (2013). Introduction: ISSVA classification. Cambridge University Press. http://assets.cambridge.org/97805218/ 48510/exce rpt/9780521848510_excerpt.pdf.

Fernandes, D. T., Elias, R. A., Santos-Silva, A. R., Vargas, P. A., \& Lopes, M. A. (2018). Benign oral vascular lesions treated by sclerotherapy with ethanolamine oleate: A retrospective study of 43 patients. Med Oral Patol Oral Cir Bucal, 23 (2), 180-e187. 10,4317 / medoral.22253.

GREENE, A. K. (2012). Current concepts of vascular anomalies. J Craniofac Surg, 23, 220-224. https://journals.lww.com/jcraniofacialsurgery/Abs tract/2012/0100 0/Current_Concepts_of_Vascular_Anomalies.54.aspx

Haggstrom, A. N., Drolet, B. A., Baselga, E., Chamlin, S. L., Garzon, M. C., Horii, K. A., Lucky, A. W., Mancini, A. J., Metry, D. W., Newell, B. , Nopper, A. J., \& Frieden, I. J. (2007) Prospective study of infantile hemangiomas: demographic, prenatal, and perinatal characteristics. J Pediatr, 150, $291-294$. 10.1016 / j.jpeds.2006.12.003.

Kaji, N., Kurita, M., Ozaki, M., Takushima, A., Harii, K., Narushima, M., \& Wakita, S (2009). Experience of sclerotherapy and embolosclerotherapy using ethanolamine oleate for vascular malformations of the head and neck. Cand J Plast Reconstr Surg Hand Surg, 43, $126-136$.

Kato, C.N.A.O., Ribeiro, M. C., do Amaral, M. B. F, Grossmann, S. M. C, de Aguiar, M. C. F., \& Mesquita, R. A. (2019) Experience with 5\% ethanolamine oleate for sclerotherapy of oral vascular anomalies: A cohort of 15 consecutive patients. J Craniomaxillofac Surg., 47 (1), 106-111. 10.1016 / j.jcms.2018.11.009.

ISSVA classification for vascular anomalies (C) (2018) http://issva.org/classification.

Johann, A. C. B. R., Aguiar, M. C. F., do Carmo, M. A. V , Gomez, R. S. , Castro, W. H., \& Mesquita, R. A.. (2005) Sclerotherapy of benign oral vascular lesion with ethanolamine oleate: An open clinical trial with 30 lesions. Oral Surg Oral Med Oral Pathol Oral Radiol Endod., 100 (5), 579-584. 10.1016 / j.jcms.2018.11.009.

Mariano, F.V., Vargas, P.A. , Coletta, R. D., \& Lopes, M. A. (2011). Sclerotherapy followed by surgery for the treatment of oral hemangioma: a report of two cases. Gen Dent., 59 (3), 121-125.

Nair, S.C. (2018). Vascular anomalies oh the head and neck region. J Maxillofac Oral Surg, 17 (1), 1-12.

Ozaki, M., Kurita, M., Kaji, N., Fujino, T., Narushima, M., Takushima, A., \& Harii, K. (2010) Efficacy and evaluation of safety of sclerosants for intramuscular venous malformations: clinical and experimental studies. Scand J Plast Reconstr Surg Hand Surg, 44, 75-87. 10.3109 / 02844310903569725.

Pereira, A. S., Shitsuka, D. M., Parreira F. J., \& Shitsuka, R. (2018). Metodologia da pesquisa científica. UFSM.

Sadick, M., Wohlgemuth, W. A., Huelse, R., Lange, B., Henzler, T., Schoenberg, S. O., \& Sadick, H. (2017) Interdisciplinary management of head and neck vascular anomalies: clinical presentation, diagnostic findings and minimal invasive therapies. Eur J Radiol Open, 14, 63-68. 10.1016/ j.ejro.2017.05.001.

Sigaux, N., Viremouneix, L., Guibaud, L., \& Bretão, P. (2015). Head and neck superficial venous malformations. Rev Stomatol Chir Maxillofac Chir Orale, $116,201-208$. 